\title{
Mechanisms of priming of pop-out: Stored representations or feature-gain modulations?
}

\author{
Hyunkyu Lee \\ University of Iowa, Iowa City, Iowa \\ Michael C. Mozer \\ University of Colorado, Boulder, Colorado \\ AND \\ Shaun P. Vecera \\ University of Iowa, Iowa City, Iowa
}

\begin{abstract}
Previous research has shown that repetition of a task-relevant attention-capturing feature facilitates popout search. This priming of pop-out effect is due to some residual memory from recent trials. We explore two possible mechanisms of priming of pop-out: a top-down attentional benefit from a memory of the previous target representation that is stored in visual short-term memory (VSTM) and a bottom-up change of attentional gains from perceptual features of the previously attended target. We manipulated participants' ability to form a memory trace in VSTM by occupying it with a distractor task and found that occupying VSTM did not interfere with priming of pop-out. We next manipulated attentional gains associated with feature values by inserting an irrelevant task between pop-out searches. We found that the color of the target from the intervening perceptual task influenced pop-out search: The current pop-out search was facilitated when the intervening task's target matched the target color of the pop-out search. These results suggest that priming of pop-out might not be due to a memory trace of the previous targets in VSTM but, rather, might be due to changes in attentional control based on priming from relatively low-level feature representations of previously attended objects.
\end{abstract}

Because of its limited computational capacity, the human brain does not fully process all of the information in a visual scene. Selective attention compensates for limitations on information processing capacity by filtering the spatiotemporal stream of information. Many accounts of attentional selection propose both bottom-up and topdown mechanisms for directing attention to objects in a visual scene. In addition to the often-discussed bottom-up and top-down attentional control processes, attention is also directed by past experience. Past experience affects our current performance across a variety of domains and experimental paradigms (see Fecteau \& Munoz, 2003; Mozer, Kinoshita, \& Shettel, 2007; Mozer, Shettel, \& Vecera, 2006), and a prior deployment of attention influences how attention will subsequently be directed.

Several experimental results have demonstrated that past experience affects visual attention in the context of visual search, in which observers search for a known target among distractors. In a series of studies, Maljkovic and Nakayama (1994, 1996, 2000; also see Hillstrom, 2000; Huang, Holcombe, \& Pashler, 2004; and Olivers \& Humphreys, 2003) investigated the influence of past experience in a simple feature search task. Observers saw three diamond-shaped items with a chip off either the left or right side. Two of the objects were the same color, and the third was a unique color. The target was the uniquely colored object in the display, and observers reported the side of the chip on this singleton target. The goal of the studies was to compare performance when the singleton target remained the same from trial to trial (e.g., a red target followed by a red target) with that when it changed (e.g., a red target followed by a green target). The results showed a clear benefit of the repetition of the singleton feature: Reaction times (RTs) fell significantly when the singleton feature had appeared on an earlier trial, and this benefit extended to five or six previous trials. That is, a same-colored target that appeared five trials earlier (trial $n-5$ ) could produce faster RTs on the current trial (trial $n$ ). Maljkovic and Nakayama (1994, 1996, 2000) called this phenomenon priming of pop-out (PoP).

PoP does not passively encode all items appearing in the visual field. In order to create a persistent memory trace, the object in the visual field must be attended (Goolsby \& Suzuki, 2001), although there is also memory for the distractors' identities. Distractor repetition also decreases RTs, although not as much as does target 
repetition (Maljkovic \& Nakayama, 1994). The memory trace formed by the previously attended object, however, does not seem to be under voluntary control. Maljkovic and Nakayama (1996) presented targets in a predictable order (e.g., green, green, red, red, green, green . . .) and found PoP independent of observers' knowledge of the sequence. Because PoP appears to be involuntary and unaffected by explicit knowledge, it is usually considered as arising from a relatively short-lived memory of the target from previous trials. Unlike other phenomena showing longer-term memory effects on attentional search (e.g., contextual cuing; Chun \& Jiang, 1998, 2003), PoP appears to be a short-term effect that lasts approximately 15-30 sec (Maljkovic \& Nakayama, 2000).

With a mass of data indicating the presence of a shortlived memory influence from previous experience on visual search, there is no consensus as to the memory system that underlies PoP. Huang et al. (2004) suggested that $\mathrm{PoP}$ arises from an episodic memory representation. They presented displays whose items varied in color and size. The target was defined as an oddball in size, and color was irrelevant for the task. Repetition of target color facilitated responses, but only if the target size was also repeated. This result implies that the memory is not independent of, but rather encodes, the configuration of features of the target (i.e., an episodic trace). The facilitation of the attentional process based on an episodic memory trace fits well with current biased competition accounts of attention (Desimone, 1998; Desimone \& Duncan, 1995; Duncan, 1996, 1998; Harter \& Aine, 1984). The memory of a previous trial's target could act to guide search in a top-down manner, possibly by maintaining a representation of the target object in visual short-term memory (VSTM). This memory trace of the previous target could be used to guide attention to a similar target on a later trial.

Another potential account of the mechanism underlying PoP has been proposed by Wolfe, Butcher, Lee, and Hyle (2003). This account is based on the guided search model (Wolfe, 1994; Wolfe, Cave, \& Franzel, 1989), which proposes that saliency at each location in the visual field is defined by the sum of feature activations at that location, where the contribution of features to the sum is modulated by a gain or weight. The gains are set in a manner appropriate for the task. For example, if the task is to search for a red vertical bar, the gains on the red and vertical feature maps should be larger than gains on the green or horizontal feature maps. In PoP, Wolfe et al. (2003) suggested that, following each trial, the gains on the feature dimensions of the current target are raised, resulting in increased gain in an activation map and reduced response latency when the target is repeated on the next trial. By this account, memory of recent trial history is encoded in feature gains (see Mozer et al., 2006).

To our knowledge, there has been no direct experimental test to distinguish the VSTM and feature-gain accounts of PoP. One line of evidence that appears at odds with a VSTM account and supports the feature-gain account is that PoP is not affected by explicit knowledge of the trial sequence. For example, Maljkovic and Nakayama (1994,
2000) reported that a predictable, alternating sequence of target colors (red, green, red, green, etc.) did not abolish the typical cost of switching target colors. Furthermore, when asked to recall previous target features, observersthe authors themselves - showed chance performance at recalling the target color from two trials back or earlier. Observers could recall features only on the most recent trial (trial $n-1)$. However, this lack of explicit memory on PoP does not necessarily rule out a VSTM representation account. First, the explicit trial sequence was presented to participants verbally, and some studies suggest that the guidance of attention by memory occurs only when it is difficult to verbalize the memory content (Olivers, Meijer, $\&$ Theeuwes, 2006). Second, because memory for previous trials was tested infrequently (after every 15-20 trials), observers' failure of explicit recollection of previous targets might be due to subthreshold representations in an explicit, episodic memory system. VSTM representations might have failed to reach threshold for explicit report because of proactive interference in the long runs of trials: Across dozens of trials, the target and distractor colors change frequently, possibly allowing proactive interference from one trial to the next to reduce explicit memory. Third, the correct response of previous trial targets' color required not only memory of the color but also binding of target color and temporal position, which might be very difficult and could easily exceed VSTM capacity.

In the present study, we directly examined whether the priming of pop-out effect arises from a memory trace in VSTM or from a feature-gain modulation. We first replicated PoP using three Landolt $\mathrm{C}$ shapes (Experiment 1); reliable PoP effects were observed for stimuli from the previous three trials. We next asked whether the PoP effect is due to a VSTM influence by using a dual-task situation in which observers held four objects in visual memory while performing a feature search task (Experiment 2); PoP appeared even when VSTM was loaded at or near capacity. Next, we conducted several control experiments testing the validity of the manipulation of VSTM and the perceptual (i.e., pop-out search) task. The VSTM manipulation in the present experiments was demanding enough to have an effect on another memory task. However, the pop-out search task showed little influence on the memory task. Finally, we demonstrated that PoP is consistent with the notion of feature-gain modulation by demonstrating a PoP effect from a secondary perceptual task that did not involve visual search (Experiment 4), suggesting that features of any attended object can be primed and can guide attention on subsequent trials.

\section{EXPERIMENT 1 Replication}

\section{Method}

Participants. Ten University of Iowa undergraduates with normal or corrected-to-normal vision volunteered in exchange for course credit.

Stimuli. The stimuli were presented on a video monitor with a black background. Red and green Landolt Cs were used. Three items (one target and two distractors) were used in a search display. Each item measured approximately $1^{\circ} \times 1^{\circ}$ of visual angle with a viewing 


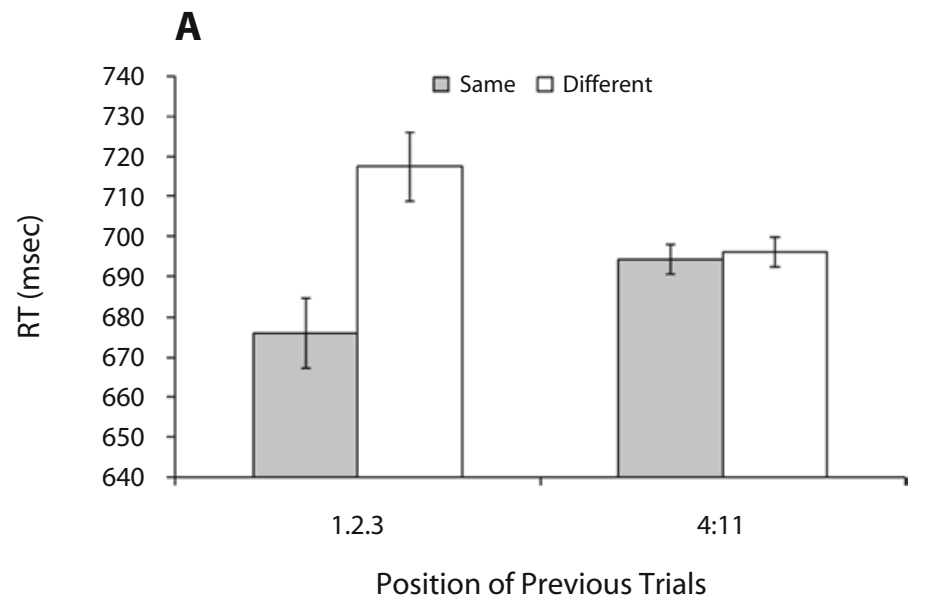

B

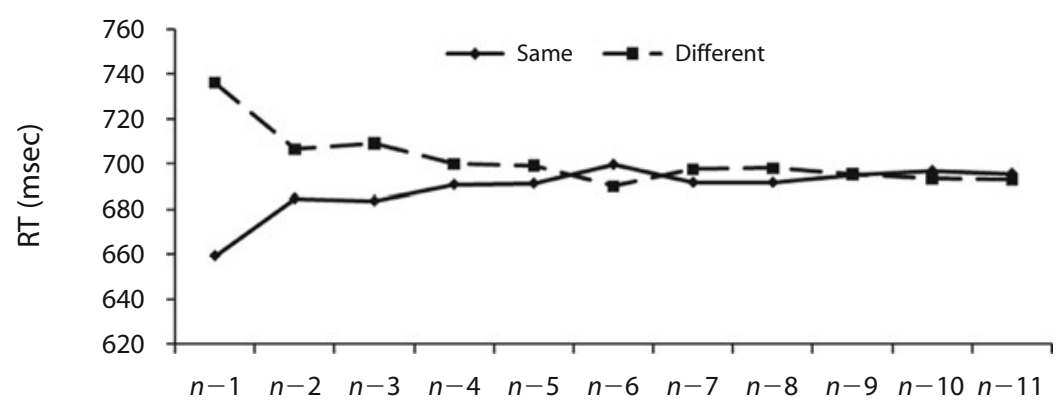

Position of Previous Trials

Figure 1. The results of Experiment 1. (A) Collapsed reaction times (RTs) for Trials 1-3 and 4-11; (B) collapsed RTs for the PoP going-back trials. Error bars represent within-subjects $95 \%$ confidence intervals on the same-repetition versus differentrepetition comparisons.

distance of $57 \mathrm{~cm}$ and had a $0.3^{\circ}$ gap either on the left or on the right side. The items were presented at randomized locations within a $4^{\mathrm{o}} \times 4^{\mathrm{o}}$ display region with a minimum center-to-center distance of $1.5^{\circ}$. The display could appear at one of four quadrants in the display with equal probability.

Procedure. Every trial started with a $500-\mathrm{msec}$ fixation cross. The search display was then presented. The target was defined by the odd color, relative to the distractors, and was equally likely to be red or green. The sequence of red or green singleton target color was random. Observers were instructed to detect the odd-color target and to report the gap location of the target as quickly and as accurately as possible. Immediately following the response, either the fixation cross or a "Wrong" feedback screen was presented for $500 \mathrm{msec}$, depending on the correctness of the response. Each observer received 20 practice trials at the beginning of the experiment and did 240 experiment trials; the practice trials were not analyzed.

\section{Results and Discussion}

The overall accuracy for the search task was $95.4 \%$. Trimming long $(>2,000-\mathrm{msec})$ and short $(<150-\mathrm{msec})$ RTs eliminated less than $1 \%$ of the data. Only trials with correct responses for the search task were analyzed. Observers' mean RTs were analyzed with a within-subjects ANOVA with previous-current target color (same vs. different) and the position of previous trials (the 3 previous trials - trials $n-1, n-2$, and $n-3-\mathrm{vs}$. the 4 th- 11 th previous trials - trials $n-4$ to $n-11$ ) as factors. The mean RTs appear in Figure 1.

An ANOVA revealed a main effect of previous-current target color $[F(1,9)=725.9, p<.001]$. Targets following a same-colored target were responded to faster than were targets following a different-colored target. The main effect of the position of previous trials was also significant $[F(1,9)=25.3, p<.001]$. Finally, the interaction between the previous-current target relation and the position of previous trials was significant $[F(1,9)=24.5, p<.001]$, indicating that the PoP was larger for the recent trials (trials $n-1$ to $n-3$ ) than for the more distant trials (trials $n-4$ to $n-11)$.

Planned comparisons showed that, on the three previous trials (trials $n-1, n-2$, and $n-3$ ), there was a significantly faster response for the target whose color matched with that of the previous target $[t(9)=5.4, p<.01]$. However, in the more distant trials (trials $n-4$ to $n-11$ ), there was no difference between the conditions of previouscurrent target color matching $[t(9)=0.5, p>.05]$.

The accuracy data were analyzed with a withinsubjects ANOVA with previous-current target color 
A

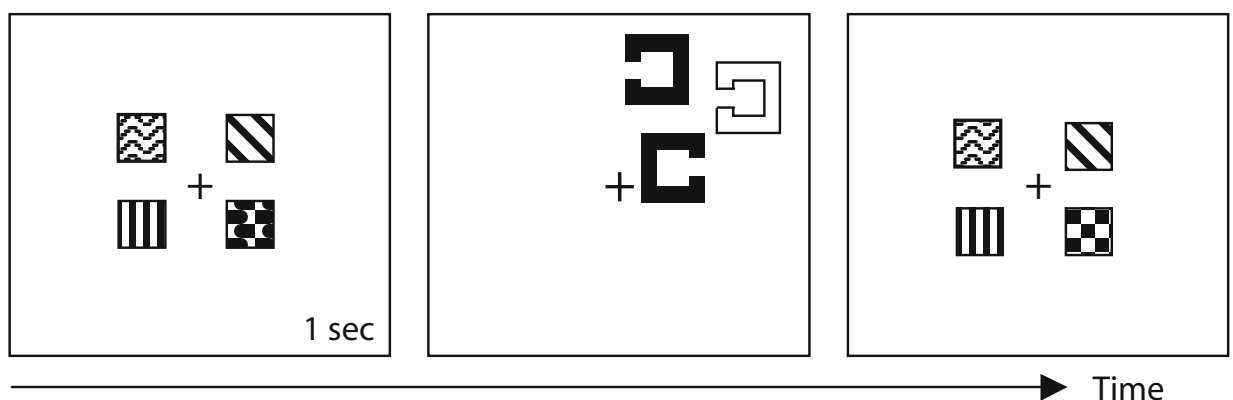

B
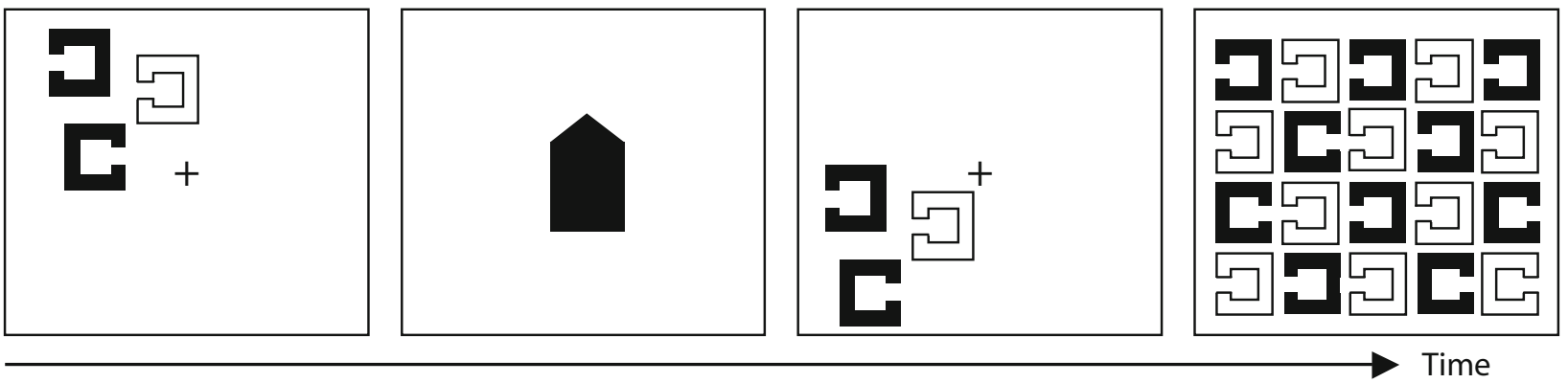

Figure 2. (A) Stimuli and order of events in Experiment 2A. Different patterns represent different colors. (B) Stimuli and order of events in Experiment 4.

(same vs. different) and the position of previous trials (the three previous trials - trials $n-1, n-2$, and $n-3$ vs. the 4 th -11 th previous trials - trials $n-4$ to $n-11$ ) as factors. There was no main effect of previous-current target color $[F(1,9)=0.4, p>.05]$. The main effect of the position of previous trials was significant $[F(1,9)=$ $6.1, p<.05]$. The interaction between the previouscurrent target relation and the position of previous trials was marginally significant $[F(1,9)=4.6, p=.06]$. Planned comparisons showed that, on the three previous trials (trials $n-1, n-2$, and $n-3$ ), accuracy was significantly higher for the target matched with the previous target for color $[t(9)=2.4, p<.05]$. However, in the more distant trials (trials $n-4$ to $n-11$ ), there was no difference between conditions of previous-current target color matching $[t(9)=0.6, p>.05]$.

These results replicate the PoP effect originally found by Maljkovic and Nakayama (1994). The previous trial affects RTs on the current trial (see Figure 1B); we found that the PoP goes back five trials, similar to the results in Maljkovic and Nakayama (1994). However, only the first three previous trials showed a significant difference between previouscurrent target color matching conditions.

\section{EXPERIMENT 2A}

In Experiment 2A, we directly asked whether the stored VSTM representations of previous targets are the primary factors for the PoP. In order to test the VSTM locus of PoP, the colors of four squares were used as a memory load and coupled with a simple pop-out search task. On each trial, observers first saw a memory array with four objects to store in visual memory. Next, a search array appeared, and the observers responded to the singleton item. Finally, a test array appeared and the observers indicated whether the objects were the same as or different from those in the memory array.

If stored VSTM representations underlie the priming effect, in the memory load condition in which VSTM was loaded at or near capacity, there should be an absent or reduced priming effect, because the memory load would prevent the current target from entering or being maintained in VSTM. Consequently, the target from the previous trial would be unable to affect search and recognition in the current trial. However, if a VSTM representation of the previous target is not the main factor of PoP, there should be a priming effect from previous target colors even when VSTM is occupied.

\section{Method}

Participants. Twenty-eight University of Iowa undergraduates with normal or corrected-to-normal vision volunteered in exchange for course credit.

Stimuli. Search arrays were identical to those in Experiment 1. Four colored squares were used as a memory array. The colors of the squares were selected at random (without replacement) from a set of six colors (white, yellow, gray, blue, purple, and brown). Each colored square subtended $0.8^{\circ} \times 0.8^{\circ}$ and was centered $1.1^{\circ}$ from fixationone above, one below, one to the left, and one to the right. 
Procedure. Figure 2A illustrates the procedure and timing. Before starting the experiment, the participants were instructed to repeat "abc" during the experiment as an articulatory suppression task. Every trial started with a $500-\mathrm{msec}$ fixation cross. The four colored squares were presented for $1,000 \mathrm{msec}$ (memory array), and the observers were instructed to remember the colors for a later test. Next, the pop-out search display was presented. The target was defined by the odd color, relative to the distractors, and was equally likely to be red or green. The observers were instructed to detect the odd-color target and to report the gap location of the target as quickly and as accurately as possible. Immediately following the response, either the fixation cross or a "Wrong" feedback screen was presented for $500 \mathrm{msec}$, depending on the correctness of the response. Then four color patches were presented as the test display. On half of the trials, this test display was identical to the study display that the observers remembered; on the other half of the trials, one color had changed to another color that was not in the study display. The observers made an unspeeded response to indicate whether the test display was identical to the study display.

There were two conditions, depending on the relation between the color of current trial's target and that of previous trial's target (previous-current target color: same or different). The observers received 20 unanalyzed practice trials at the beginning of experiment, followed by 240 experiment trials.

\section{Results and Discussion}

The data from 8 participants were dropped because of low performance (below $60 \%$ on the memory performance or search performance). ${ }^{1}$ The overall accuracy was $94.3 \%$ for the search task and $74.6 \%$ for the changedetection task. Trimming long $(>2,000-\mathrm{msec})$ and short $(<150$-msec) RTs eliminated less than $1 \%$ of the data. Only trials with correct responses for both search and memory tasks were analyzed. The observers' mean RTs were analyzed with a within-subjects ANOVA with previous-current target color (same vs. different) and the position of previous trials (the three previous trialstrials $n-1, n-2$, and $n-3$ - vs. the 4 th to 11 th previous trials - trials $n-4$ to $n-11$ ) as factors. The mean RTs appear in Figure 3A.

A

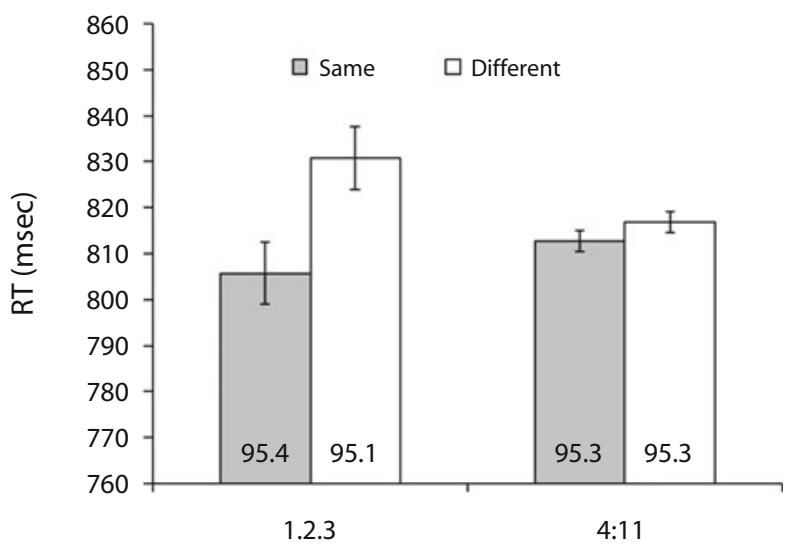

Position of Previous Trials
We found a main effect of previous-current target color $[F(1,19)=18.3, p<.05]$. Same-colored targets were responded to faster than were different-colored targets. The main effect of the position of previous trials was significant $[F(1,19)=4.8, p<.05]$. The interaction between the previous-current target relation and the position of previous trials was also significant $[F(1,19)=9.9, p<$ .05]. Planned comparisons showed that, on the three previous trials (trials $n-1, n-2$, and $n-3$ ), there was a significantly faster response for the target matched with the previous target for color $[t(19)=3.9, p<.05]$. However, in the more distant trials (trials $n-4$ to $n-11$ ), there was no difference between the conditions of previous-current target color matching $[t(19)=1.9, p>.05]$. The accuracy data were not significant in any conditions.

These results replicate the PoP effect originally found by Maljkovic and Nakayama (1994) and further suggest that the priming of the pop-out target from one trial to the next is not due to a VSTM representation of the previous trial's target. Loading VSTM does not appear to affect PoP.

Although the present results suggest that PoP was not abolished when VSTM was loaded, the amount of priming was less strong than the priming effect found in Experiment 1 ( $25 \mathrm{msec}$ and $41 \mathrm{msec}$, respectively). It is possible that the representation of previous targets stored in VSTM affects the priming effect of subsequent targets, but loading VSTM only slows or weakens the priming effect. However, Experiment 2A also differed from Experiment 1 in timing. The intertrial interval in Experiment 2A was approximately double that in Experiment 1, because of the presentation of the memory array, the test array, and the response. It is also possible that the colors of the to-be-remembered objects interfered with the feature representations of the search array stimuli, thereby diluting the PoP effect.

In order to determine whether the reduced priming effect that we observed in Experiment 2A was caused by

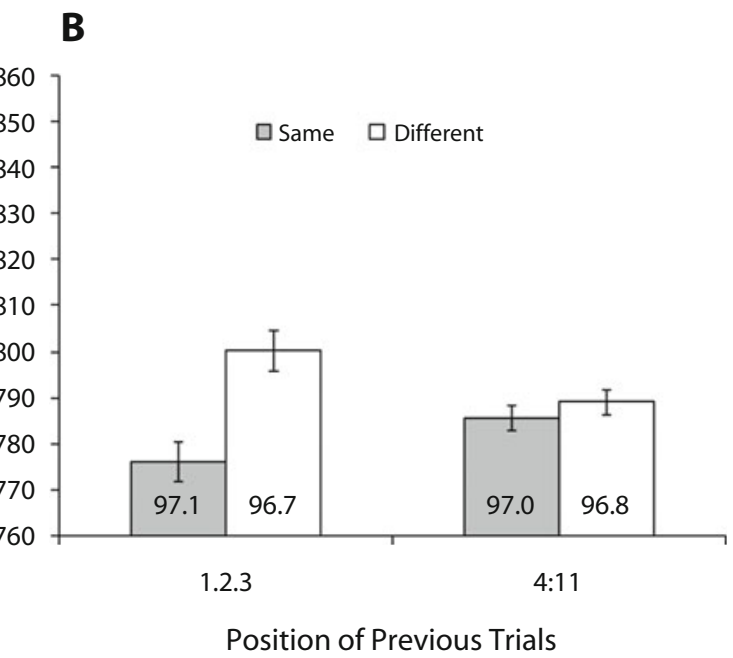

Figure 3. (A) The results of Experiment 2A. (B) The results of Experiment 2B. Error bars represent within-subjects 95\% confidence intervals on the same-repetition versus different-repetition comparisons. The number on each bar represents the accuracy of each condition. RT, reaction time. 
the VSTM load or by the increased time delay used in our procedure, in Experiment 2B, we presented the participants with the same timing as that used in Experiment 2A. In Experiment 2B, however, the participants did not need to store the colored squares in VSTM. If a VSTM load reduces the priming effects observed originally by Maljkovic and Nakayama (1994), performance in this no-load experiment should produce priming effects significantly larger than those observed in Experiment 2A. In contrast, if the increased timing due to interspersing the change detection task with search trials reduced the PoP effect, the results from the present no-load experiment should be similar to those observed in Experiment 2A.

\section{EXPERIMENT 2B}

\section{Method}

Participants. Twenty University of Iowa undergraduates with normal or corrected-to-normal vision volunteered in exchange for course credit

Stimuli. The displays were identical to those in Experiment 2A.

Procedure. The procedure used in Experiment 2B was identical to that used in Experiment 2A but with two exceptions. In Experiment $2 \mathrm{~B}$, the memory array and the test array were replaced with a fixation cross and a blank display, respectively. The blank display was presented for $1,635 \mathrm{msec}$, which was the mean response duration for memory displays for Experiment 2A.

\section{Results and Discussion}

The general accuracy was $96.9 \%$ for the search performance, and trimming long $(>2,000$-msec $)$ or short $(<150$-msec) RTs eliminated less than $1 \%$ of the data. As in Experiment 2A, observers' mean RTs were analyzed with a within-subjects ANOVA with previous-current target color (same vs. different) and the position of previous trials (the previous three trials vs. the 4th-11th previous trials) as factors. The mean RTs appear in Figure 3B.

As in the previous experiments, we again found a main effect of previous-current target color $[F(1,19)=39.9$, $p<.05]$. Targets following a same-colored target were responded to faster than were targets following a differentcolored target. The main effect of the position of previous trials was not significant $[F(1,19)=1.8, p>.05]$. The interaction between previous-current target color and the position of previous trials was significant $[F(1,19)=14.6$, $p<.05]$. Planned comparisons showed that, in the three previous trials (trials $n-1, n-2$, and $n-3$ ), there were faster responses when the current target's color matched that of the previous target than when these colors were mismatched $[t(19)=5.8, p<.05]$. However, in the more distant trials, there was no difference between the conditions of previous-current target color matching $[t(19)=$ $1.3, p>.05]$. The accuracy data were not significant across any of the conditions.

To examine the magnitude of the priming effect across load and no-load conditions, we conducted an additional mixed-model ANOVA, with previous-current target color (same vs. different) on the three previous trials as a within-subjects factor and load (Experiment 2Aload-vs. Experiment 2B-no load) as a between- subject's factor. There was no main effect of experiment $[F(1,38)=0.48, p>.1]$. However, there was a numeric difference, in that Experiment 2B (no-load condition) showed faster RTs $(788 \mathrm{msec}$ ) than did Experiment 2A (load condition, $818 \mathrm{msec}$ ). There was a main effect of previous-current target color condition $[F(1,38)=40.5$, $p<.01]$. Most important, the interaction between experiment and previous-current target color was not significant $[F(1,38)<1$, n.s.]. In short, the size of the priming effects was similar across Experiments $2 \mathrm{~A}$ and $2 \mathrm{~B}$, suggesting that a VSTM load does not affect the magnitude of priming in a pop-out search. VSTM does not appear to be the source of the priming effects observed in the PoP phenomenon.

With timing identical to that in Experiment 2A, Experiment $2 \mathrm{~B}$ showed an almost identical priming effect without VSTM load. These results suggest that the reduced priming effect in Experiment 2A relative to those found in previous reports was because of the time delay between trials. Again, the results of Experiments 2A and 2B, taken together, suggest that VSTM is not responsible for the PoP effect. A feature search can be primed even when VSTM is occupied with four objects.

The present experiments have demonstrated that VSTM load does not affect the PoP effect, suggesting that PoP might be based on the feature-level attentionalgain change rather than on the memory representation in VSTM. However, this conclusion is based on the assumption that there is no VSTM capacity remaining during the load condition. If this assumption is incorrect and another item - namely, the pop-out target-entered VSTM relatively easily, the results of Experiment 2 could be due to storage of the pop-out target in visual memory. Although VSTM capacity for colored squares is typically estimated at 3-4 objects (e.g., Luck \& Vogel, 1997), it is possible that there was still enough room in VSTM to squeeze in the pop-out target, because the target's color and shape did not overlap with, and possibly did not interfere with, the items already in VSTM.

In order to examine these possibilities, we conducted a series of control experiments. The aims of these experiments were to ensure (1) that holding the colors of four squares in VSTM filled memory to capacity and impaired performance on another memory task and (2) that the target of a pop-out search does not automatically enter visual memory and interfere with items already stored there.

In Experiments 3A and 3B, we asked observers to perform two memory tasks: the standard four-color memory task used in Experiment 2 and a memory task to remember the location of the gap on a singleton target. The gap memory task used the same pop-out search displays used in Experiment 2. On some trials, the participants had to remember both the gap location of a singleton and the colors of four squares in VSTM (the dual-task condition). On other trials, the participants remembered either the colors of the four squares (Experiment 3A) or the singleton's gap location (Experiment 3B) but not both. If there was room left in VSTM for the pop-out target when memory was loaded with four colors, the participants should remember 
A

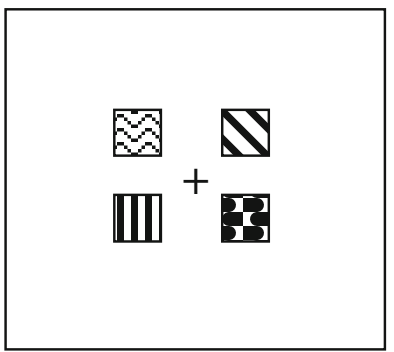

$1 \mathrm{sec}$

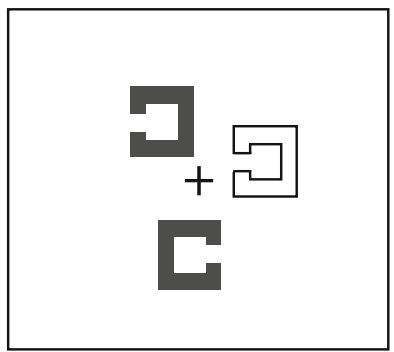

$1 \mathrm{sec}$

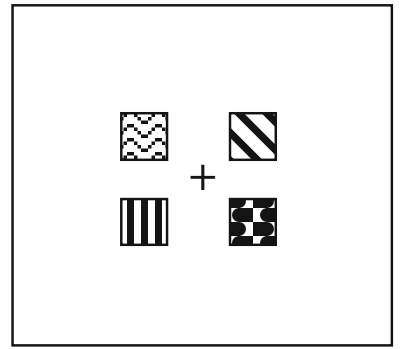

Color Memory Test

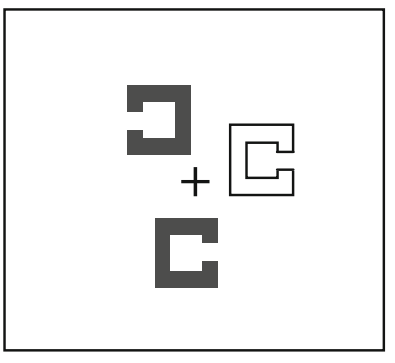

Shape Memory Test

B

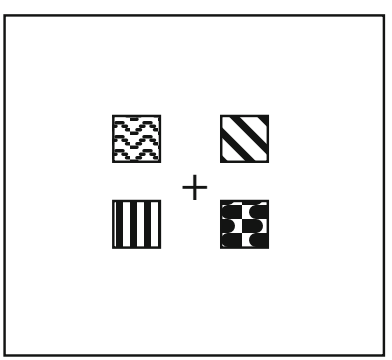

$1 \mathrm{sec}$

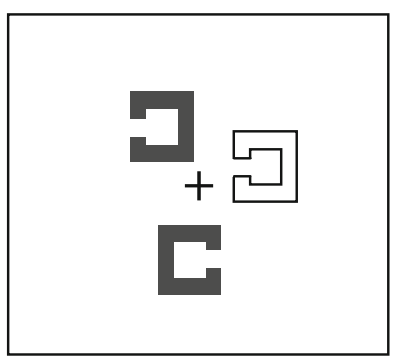

Pop-Out Display

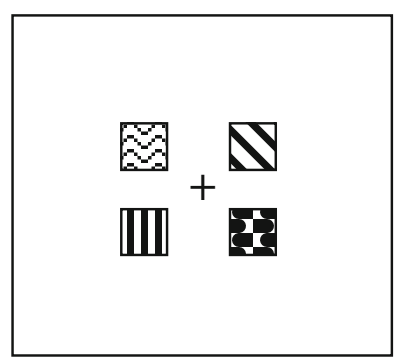

Color Memory Test

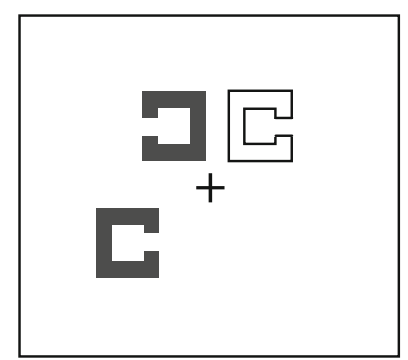

Pop-Out Display

Time

Figure 4. (A) Stimuli and order of events in Experiments $3 A$ and $3 B$. In the dual-task condition, observers were instructed to remember four colors and the gap location of a singleton target. In the single-task condition of Experiment $3 \mathrm{~A}$, observers were instructed to remember only the four colors. In the single-task condition of Experiment $3 B$, observers were instructed to remember only the gap location of a singleton target. (B) Stimuli and order of events in Experiment $3 \mathrm{C}$. In the dual-task condition, observers were instructed to remember four colors and to perform a singleton target discrimination task. In the single-task condition, observers were instructed to remember only the four colors.

the singleton's gap as accurately when it is remembered alone as when it is remembered along with the four coloreds. However, if holding the colors of four squares fully occupies VSTM capacity, the participants should be more accurate in reporting the singleton's gap location when memory is not loaded with the colors than when memory is loaded with them.

Finally, we asked whether the target of a pop-out search automatically enters visual memory. In Experiment 3C, we asked observers to perform a pop-out search while they were holding the four colors in VSTM. The pop-out search was identical to that used in Experiment 2. The observers attended and responded to the pop-out target without the explicit goal of remembering it. On some trials, the observers performed the pop-out search while holding the four colors in VSTM (the dual-task condition). On other trials, the observers only remembered the colors of four squares (the single-task condition). If the pop-out target automatically squeezes itself into VSTM, there should be an accuracy drop in memory recall in the dual-task condition relative to the single-task condition, in which observers only performed the visual memory task.

\section{EXPERIMENTS 3A-3C}

\section{Method}

Participants. For each experiment, 10 University of Iowa undergraduates with normal or corrected-to-normal vision volunteered in exchange for course credit.

Stimuli. Stimuli were identical to those in Experiment 2A with one exception. The pop-out display was always presented at the center instead of at one of four randomly selected quadrants.

Procedure. Figure 4 illustrates the procedure and timing of Experiments $3 \mathrm{~A}, 3 \mathrm{~B}$, and $3 \mathrm{C}$. Before starting the experiment, the observers were instructed to recite the alphabet during the experiment as an articulatory suppression task. Every trial started with a 500-msec fixation cross. In Experiment 3A, four colored squares were presented for $1,000 \mathrm{msec}$ (memory array). The observers were instructed to remember the colors for a later test. Next, the pop-out display was presented for $1,000 \mathrm{msec}$. The target was defined by the odd color, relative to the distractors, and was equally likely to be red or green. In some trials, the observers were instructed to remember the gap location of the target (the dual-task condition). Then, four color patches were presented as the color-test display. On half of the trials, this color test display was identical to the memory array that the observers remembered; on the other half of the trials, one color had changed to another color that was not in memory array. The observers made an unspeeded response to indicate whether the color-test display was identical to the memory 
Table 1

Results of Experiments 3A, 3B, and 3C

\begin{tabular}{cccccc}
\hline & \multicolumn{2}{c}{ Dual-Task Condition Rule } & & \multicolumn{2}{c}{ Single-Task Condition Rule } \\
\cline { 2 - 3 } \cline { 5 - 6 } Experiment & Color Memory & Gap Memory & & Color Memory & Gap Memory \\
\hline 3A & 71.7 & 80.7 & & 85.0 & - \\
3B & 67.8 & 72.3 & & - & 90.8 \\
3C & 76.8 & - & & 82.7 & - \\
\hline
\end{tabular}

array. Following the response, the pop-out display was presented as the gap memory test display. On half of the trials, the gap location of the target was changed. In the dual-task condition, the observers made an unspeeded response to indicate whether the gap location was identical to the original location. In the single-task condition, observers ignored the gap test display. The gap test display was presented either until a response was made (in the dual-task condition) or for $1 \mathrm{sec}$ (in the single-task condition). For each response, either the fixation cross or a "Wrong" feedback screen was presented for $500 \mathrm{msec}$, depending on the correctness of the response.

Experiments 3B and 3C were identical to Experiment 3A, except for the task instructions. In Experiment 3B, in the single-task condition, the observers ignored the colors and remembered the target gap location only. The color-test display was presented either until a response was made (in the dual-task condition) or for $1 \mathrm{sec}$ (in the single-task condition). In Experiment 3C, the observers were instructed to report the gap location of the target as quickly and as accurately as possible (i.e., a pop-out search task instead of the target gap memory task). In the single-task condition, the observers ignored the pop-out display and remembered the colors of only the four squares. The pop-out display was presented either until a response was made (in the dual-task condition) or for $1 \mathrm{sec}$ (singletask condition)

\section{Results and Discussion}

The mean accuracies for color memory and gap memory (or gap localization) appear in Table 1. In Experiment $3 \mathrm{~A}$, we conducted a planned comparison of the color memory accuracies between the single- and dualtask conditions. The results revealed a significant accuracy difference between single- $(85 \%)$ and dual- $(71.7 \%)$ task conditions $[t(9)=5.6, p<.01]$. The gap memory accuracy in the dual-task condition was $80.7 \%$. These findings indicate that the colors of the four squares filled VSTM adequately. When the observers needed to remember the gap on a singleton target for a later report, memory for the colors declined significantly, indicating that there was insufficient room in VSTM for both the colors of the four squares and the singleton target.

In Experiment 3B, we conducted a planned comparison of the gap memory accuracies between single- and dualtask conditions. The results showed that there was a significant accuracy difference between the single- $(90.8 \%)$ and dual- $(72.3 \%)$ task conditions $[t(9)=6.6, p<.01]$. The color memory accuracy in the dual-task condition was $67.8 \%$. These findings show that, when the observers needed to remember the colors of four squares for a later report, memory for the gap on a singleton declined significantly, indicating that there was insufficient room in VSTM for both the colors of the four squares and the singleton target.

In Experiment 3C, we conducted a planned comparison of the color memory accuracies between dual- and single- task conditions. The results showed that there was a small, but significant, accuracy difference between the single$(82.7 \%)$ and dual- $(76.8 \%)$ task conditions $[t(9)=2.6$, $p<.05]$. This latter result initially appears to suggest that the targets of a pop-out search automatically enter VSTM and interfere with objects already stored in memory. However, it is also possible that the accuracy decrement that we observed in Experiment 3C was due to a general dual-task decrement that arose because the observers were performing two tasks (color memory and singleton search), not because the singleton item entered VSTM.

In order to test whether the color memory accuracy drop in the dual-task condition was due to the pop-out target's entering memory or due to more general dual-task interference, we asked whether the memory interference that we observed in Experiment 3A differed from that observed in Experiment 3C. Experiment 3A represents a situation in which a new item in memory (the pop-out target) interferes with the storage and maintenance of the colors of the four squares. If the interference observed in Experiment 3C was due to memory interference, we should observe the same accuracy decrement between Experiments $3 \mathrm{~A}$ and $3 \mathrm{C}$. However, if the accuracy drop in Experiment $3 \mathrm{C}$ was due to a more general dual-task decrement, the accuracy drop in Experiment $3 \mathrm{C}$ should be smaller than that observed in Experiment 3A.

To address this issue, we conducted an additional mixed-model ANOVA, with load condition (dual task vs. single task) as a within-subjects factor and memory condition (Experiment 3A-gap memory-vs. Experiment 3C - gap localization) as a between-subjects factor. There was no main effect of memory condition $[F(1,18)=$ $0.195, p>.05]$. The main effect of load was significant $[F(1,18)=34.8, p<.01]$. Most important, there was a significant interaction between load and memory condition $[F(1,18)=5.2, p<.05]$. The difference in color memory accuracy between the dual- and single-task conditions was significantly larger in the gap memory dualtask condition (Experiment 3A, 13.2\%) than in the gap localization dual-task condition (Experiment 3C, 5.8\%). These findings suggest that the small performance drop in Experiment 3C was likely due to a general dual-task decrement and not to a pop-out target entering VSTM (as was required in Experiment 3A).

The results of Experiments 3A and 3B demonstrated that the VSTM manipulation used in Experiment 2 (remembering four colors) filled VSTM to capacity or near capacity. Asking the observers to remember one gap location in addition to the four colors drastically impaired the four-color memory performance (Experiment 3A). 
Asking the observers to remember four colors in addition to one gap location also drastically impaired the gap memory performance (Experiment 3B). Experiment 3C demonstrated that the target of the perceptual task did not appear to be stored automatically in VSTM. Instead, these results showed that responding to the perceptual feature of the singleton item while maintaining four colors did not impair VSTM performance as much as did storing multiple items in VSTM (Experiment 3A). The lower VSTM accuracies when the observers held the four colors in memory and performed pop-out search in Experiment 3C were likely due to dual-task interference instead of to interference from memory of perceptual target squeezed into VSTM.

The results of Experiments 1-3 replicated the PoP phenomenon and suggest that this priming effect can be observed when VSTM is occupied. PoP, therefore, appears to arise from changes to feature-based weights or gains, not from the representation of previous targets in VSTM. In our final experiment, we sought additional support for a feature-based gain account by asking whether a feature search could be primed by a simple perceptual task that did not require VSTM but did involve visual features that were shared between a prime display and a feature search display.

\section{EXPERIMENT 4}

Our previous experiments demonstrated that loading VSTM with the colors of four squares did not affect the PoP effect. If stored VSTM representations of previous targets were not causing the priming effect on pop-out search, then the other plausible mechanism of PoP is gain modulation of features contributing to the activity map (Wolfe et al., 2003). To evaluate the contribution of feature-gain modulation to PoP, we interleaved the popout search task with a simple shape-discrimination task that did not require any attentional selection. For the shape-discrimination task, one red or green house-shaped object (see Figure 2B) was presented, and the observers' task was to indicate the direction of the pointed end of the shape. This task did not require search, because there was only one object. Also, the shape-discrimination task did not involve color as a task-relevant feature. However, attending to the object's shape should also select the object's color, permitting the representation of color features. Furthermore, because this simple shape-discrimination task was not related to the pop-out search and because the stimuli for the tasks differed, performance on the shapediscrimination task target should not influence any episodic memory trace of previous pop-out targets. Thus, if PoP is due to stimulus or task similarity, we would expect to see little priming from one trial to the next (i.e., from a house stimulus to the search task). In contrast, if PoP is due to increasing the attentional gains of the simple features of attended shapes, we should see trial-to-trial priming because a simple feature-color-was shared across the two tasks. That is, when attending to the shape of the house-shaped object in the shape-discrimination task, its color would also be selected, allowing color information to be primed and to influence the subsequent search display.

\section{Method}

Participants. Thirty University of Iowa undergraduates with normal or corrected-to-normal vision volunteered in exchange for course credit.

Stimuli. Stimuli for the pop-out search were identical to those in Experiment 2. For the shape-discrimination task, either a red or a green house-shaped object was used. The house-shaped object consisted of a rectangle, which subtended $2.2^{\circ} \times 2^{\circ}$, and a triangular shape, which subtended $2.2^{\circ}$ wide $\times 0.2^{\circ}$ tall, pointing either upward or downward.

Procedure. Two pop-out search tasks sandwiched a shapediscrimination task, and this sandwich comprised one trial (see Figure 2B). Every trial started with a 1-sec fixation point followed by pop-out search array (as in Experiment 2). After a 500 -msec feedback screen regarding search performance, the discrimination task object was presented at the center of screen until a response was made. The observers were instructed to report the direction of the pointed end of the object. After $500 \mathrm{msec}$ of feedback for the shape-discrimination task, another search array was presented. After $500 \mathrm{msec}$ feedback for the second search task, a mask display appeared; the mask was composed of a mixture of target and distractor items and extended across the area occupied by the search array. The mask was presented for $1 \mathrm{sec}$.

The main interest of this experiment was the RT to the second search task based on the color of the target in the first search task and the color of the shape-discrimination task stimulus. We used a factorial manipulation of the relation between the status of the search target colors between the first and second search displays (the target colors were the same or different) and between that of the shape-discrimination task target color and that of the second search target color (the same or a different color). The observers received 20 unanalyzed practice trials at the beginning of experiment, followed by 256 experimental trials.

\section{Results and Discussion}

The overall accuracies were $97.6 \%$ for the search task and $98.4 \%$ for the shape-discrimination task. Trimming long $(>2,000-\mathrm{msec})$ or short $(<150-\mathrm{msec})$ RTs eliminated less than $1 \%$ of the data. Observers' mean RTs to the second search display were analyzed with a within-subjects ANOVA with target color compared with the first search array (same vs. different) and the shape-discrimination task target color (same as vs. different from the search target color) as factors. The mean RTs appear in Figure 5. There was a main effect of search target color $[F(1,29)=$ $36.3, p<.01]$. Singleton targets following a singleton target of the same color were responded to faster than singleton targets following a singleton target of a different color. The main effect of the shape-discrimination task's target color was also significant $[F(1,29)=11.8, p<.01]$, with faster responses when the house-shaped object had the same color as the singleton target. The interaction between the colors of the search and shape-discrimination targets was not significant $[F(1,29)=1.4, p>.05]$. The accuracies for the search and shape-discrimination tasks were high (over 97\%) and were not significant in any condition.

The influence of the first pop-out search target on the subsequent search task was present regardless of the in- 


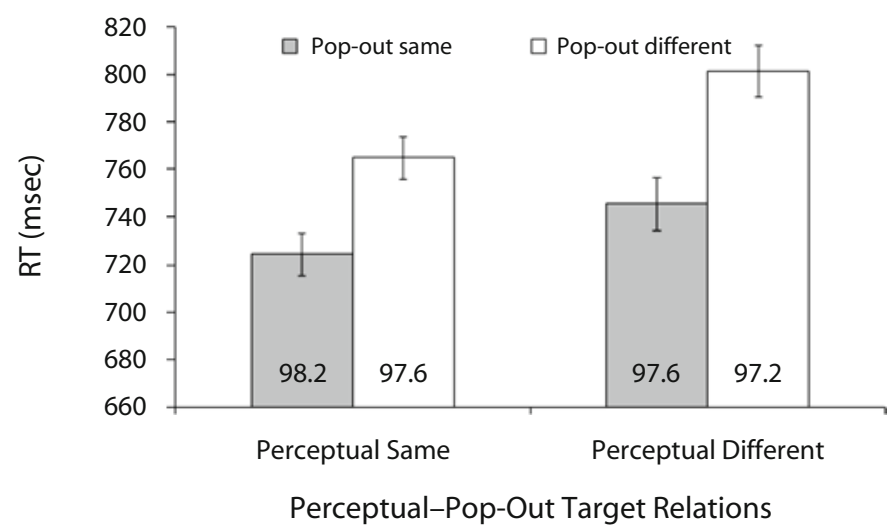

\begin{abstract}
Figure 5. The results of Experiment 4. Error bars represent withinsubjects $95 \%$ confidence intervals on the pop-out target same-color repetition versus different-color repetition comparisons. The number on each bar represents the accuracy of each condition. RT, reaction time.
\end{abstract}

termediate shape-discrimination task target color. Planned comparisons revealed that there was a significant effect of the first search task's target color in both intermediate shape-discrimination task target color conditions [same, $t(29)=4.3, p<.01$; different, $t(29)=4.9, p<.01]$. The influence of the shape-discrimination task on the subsequent search task was present, regardless of the previous search display's target color. Planned comparisons revealed that there was a marginally significant effect of the shape-discrimination task's target color when successive search displays had same-colored targets $[t(29)=$ $1.9, p=.07]$. There was also a significant effect of the shape-discrimination task's target color when successive search displays had different-colored targets $[t(29)=3.7$, $p<.01]$.

Although we did not find an interaction between the color of the previous pop-out target and that of the perceptual task, there was a trend toward this interaction. Specifically, there was a tendency for faster responses when the previous pop-out target color matched the current pop-out target color than when the perceptual-task target color matched the current pop-out target color. This finding suggests that the color of the house-shaped object did not produce as large of a feature-gain change as did the color of the previous pop-out target. The reason for this trend is likely that there is some priming from the shapes of the stimuli. Although the colors were the same for the pop-out targets and the house-shaped object, the shapes of these stimuli differed. There might have been shape and color priming from the previous pop-out search on the current pop-out search, but there was only color priming from the perceptual task on the current pop-out search task. We would hasten to add, however, that any shape priming was rather small, because the relevant interaction was not statistically significant, even after we added additional participants to the present experiment. The primary conclusion that we draw from Experiment 4 is that feature priming can occur between two tasks that involve stimuli that are only superficially similar (i.e., similar on one feature, such as color in the present experiment).

\section{GENERAL DISCUSSION}

The goal of these experiments was to investigate the locus of the PoP search. There are at least two different accounts that could explain the guidance by PoP: one based on a VSTM representation of previously selected targets and another based on priming of features of previously attended targets. Our results indicate that the benefit from previous trials does not come from a VSTM representation of the previous trial's target; instead, our results are more consistent with the notion that $\mathrm{PoP}$ arises from attentional-gain modulation of features belonging to targets on recent trials. In Experiments 2A and 2B, interrupting memory capacity with another memory load did not change the pattern of PoP. Experiment 4 showed that taskirrelevant features that belong to a single attended shape can affect pop-out search efficiency. Because the shapediscrimination task targets were perceptually distinct from the search targets and because there was no explicit goal of remembering the target, the priming effect on pop-out search might not be from a VSTM representation in memory, but from some type of feature-level trace. Control experiments confirmed that the VSTM load used in the present study filled in the VSTM capacity and did not allow another object to squeeze into VSTM (Experiments 3A and $3 \mathrm{~B}$ ). Also, the target representation of the perceptual task was not stored in VSTM (Experiment 3C).

Our results are in agreement with the results by Wolfe et al. (2003) suggesting that carryover effects from previous trials are due to the adjustment of weights (i.e., feature gains). For example, Wolfe et al. (2003) compared RTs of various blocked conditions. There were three types of feature searches: search for a pop-out in color, size, or orientation. In one condition, all the feature searches were blocked (the blocked condition); in another condi- 
tion, all three feature searches were intermixed in one block. The mixed condition had two difficulties of color search: easy and hard. In the easy color condition, the target and distractors were either red or green, and, in the hard color condition, the target and distractors were either red or orange, which were more difficult to discriminate. Response RTs were the fastest in the blocked condition; most important, the RTs in the fully mixed easy condition were shorter than those in the fully mixed hard condition. Wolfe et al. (2003) reasoned that the blocked condition generates the fastest RT because it is possible to increase attentional gains along only one feature dimension. In other words, there is a strong signal on one dimension and little interference from the other dimensions, leading to fast RTs. In the fully mixed condition, every feature is equally likely to be a target feature. So, every dimension receives comparable attentional-gain modulations, and the signal-to-noise ratio is roughly equal for all target types. In the fully mixed hard condition (e.g., a red target among orange distractors), the color dimension receives extra weighting - unlike in the fully mixed easy condition - in order to boost color feature performance and to reduce the noise. As a result, when a target with an orientation or size singleton appears, responses are slowed, because of the added noise from the color dimension (see also Muller, Heller, \& Ziegler, 1995, and Muller, Reimann, \& Krummenacher, 2003, for a dimensional weighting account).

Our results can be understood in the same architecture as that in Wolfe et al. (2003), such that, when the display is presented, a set of basic attributes along different dimensions (e.g., color, orientation, size) are extracted in parallel. The activation of each dimension is computed in parallel, and then these signals are summed onto a master map. Attention is directed to the location with the most activation on the master map (Wolfe, 1994, 1998). Our results suggest that, in attending to a target, the target's features will be detected and (implicitly) deemed salient on subsequent trials. These salient features might alter the activation across a master map of locations, allowing attention to visit locations containing features that match the features of the previous target.

Priming effects from task-irrelevant features and the interaction between task-relevant and task-irrelevant feature repetition (e.g., Huang et al., 2004) can be easily explained in the same architecture. Once attended, all features of the attended item might get processed and change the corresponding attentional gains associated with the object's features, not only of the task-relevant feature, but also of the task-irrelevant features. In short, attentional gains might be altered on the basis of objects. That is, the features of an attended object might be bound together to form an object, and changes to any of the features in a later display might minimize priming because the object has changed. For example, repetition of a big red target coactivates features for red and big. If a small red target appears after the repetition of a big red target, red carries weakly over to the next trial, if at all, because the other feature (size) mismatches; the targets across these two trials are treated differently because they are different objects (i.e., one is large and the other small).

Interestingly, although attentional-gain modulations may underlie PoP, these gain modulations are likely modulated by task relevance (Fecteau, 2007; Folk \& Remington, 2008). Fecteau presented observers with displays containing two feature singletons: a color singleton and a shape singleton. One of these singletons was task relevant and was cued at the beginning of the trial. PoP was found only for singletons that were task relevant. That is, if a color singleton was selected on trial $n-1$, features of this singleton affected responses on trial $n$ only if the color singleton was again selected. Features from the taskirrelevant shape singleton in trial $n-1$ did not affect responses to targets in trial $n$, even when the task on trial $n$ involved selecting the shape singleton.

On the surface, Fecteau's (2007) result appears at odds with those of our Experiment 4, in which we found that a shape-discrimination task could prime pop-out search. However, the shape-discrimination task used in Experiment 4 contained a single shape that, itself, would have popped out of the display. Attentional control (à la Folk, Remington, \& Johnston, 1992) might be set broadly to search for a singleton target, allowing for priming to occur between the shape-discrimination task and the pop-out search task, despite the differences between these two tasks. Because the two singletons in Fecteau's displays would have competed for attention, a more precise control setting (e.g., search for a color singleton) might have been used to guide attention and produce priming effects.

The present study focused on the mechanisms of a target-driven intertrial effect. However, there are also intertrial effects driven by distractors, known as the colorsalience aftereffect (Goolsby, Grabowecky, \& Suzuki, 2005 ) and the distractor preview effect (Ariga \& Kawahara, 2004; Lleras, Kawahara, Wan, \& Ariga, 2008). In those studies, the color oddball pop-out task trials were interspersed with target-absent trials, which consisted of three same-colored items (i.e., all distractors). The popout task RTs were analyzed by the item color of the previous target-absent trial. The results showed that, when the target color of the current pop-out task was the same as the item color of the previous target-absent trial, their RTs were about 50-100 msec slower than when the distractor color of the current pop-out search task was the same as the item color of the previous target-absent trial.

Consistent with the present study, the color salience and distractor preview aftereffects can be understood as attentional phenomena that reflect a change of feature gains based on previous experience (Lleras et al., 2008). The color of items in target-absent trials receives a negative attentional gain, because the search for a target failed. If the subsequent pop-out target shares a color with the previous distractors, the pop-out target performance gets impaired because of the attentional setting against the target color. In the same vein, if the subsequent distractors share a color with the previous distractors, the pop-out target performance gets enhanced because of the attentional setting against the distractor color. Lleras 
et al. showed that the distractor preview aftereffect is present only if the task requires focal attention (see also Goolsby et al., 2005, and Ariga \& Kawahara, 2004, for a perceptual-suppression account of this aftereffect). The difference between the present results and these aftereffects might be that the present results showed a positive attentional bias to the attended item and the aftereffect tasks show a negative attentional bias to the attended item.

The present results are broadly consistent with our recent efforts to provide a computational account of attentional priming (Mozer et al., 2006). Our account explains attentional priming phenomena by proposing that the visual system creates a probabilistic model of the environment based on past experience (i.e., previous trials). The environmental model updates the probabilities for certain target occurrences on the basis of previous trials; that is, following a red target, the environmental model is updated to expect that red targets are more probable than other targets, much as an attentional-gain account proposes. The other, task-irrelevant features of the target are also updated in the environmental model, which is necessary to allow our account to explain results from studies in which the task-irrelevant features of a target facilitate RTs (Huang et al., 2004), but the updating of these features is dependent on the value of the task-relevant feature.

One relevant question concerns the mechanism that allows a previous trial to influence attention in the current trial. Perceptual-level gain modulations probably do not influence attention on the basis of perceptual sensitivity; that is, a primed target color does not make that color more perceptible and, therefore, more likely to be attended. Rather, PoP based on attentional-gain modulations can be understood as acting to guide or prioritize attention toward the primed feature (see Huang et al., 2004). For example, other work from our lab sandwiched a vernier offset discrimination task between two pop-out searches (Lee \& Vecera, 2007). The color of the vernier discrimination task's target could be either the same as or different from the previous search target color. The results showed that the repetition of the target color did not change vernier acuity performance (a test of perceptual sensitivity). Thus, PoP, mediated by feature priming, may prioritize the deployment of attention but not affect the quality of perception.

In summary, the present experiments suggest that the implicit influence from previous trials is achieved via attentional-gain modulations. The implicit priming based on attentional-gain modulations appears to be different from attentional guidance based on a visual-memory representation (e.g., Bundesen, 1990; Desimone \& Duncan, 1995).

\section{AUTHOR NOTE}

This research was made possible by National Science Foundation Grant 03-39171 awarded to M.C.M. and S.P.V. Correspondence concerning this article should be addressed to S. P. Vecera, Department of Psychology, University of Iowa, E11 Seashore Hall, Iowa City, IA 52242-1407 (e-mail: shaun-vecera@uiowa.edu).

\section{REFERENCES}

Ariga, A., \& KaWahara, J. (2004). The perceptual and cognitive distractor-previewing effect. Journal of Vision, 4(10, Art. 5), 891-903. doi: $10.1167 / 4.10 .5$

Bundesen, C. (1990). A theory of visual attention. Psychological Review, 97, 523-547.

Chun, M. M., \& JiAng, Y. (1998). Contextual cuing: Implicit learning and memory of visual context guides spatial attention. Cognitive Psychology, 36, 28-71.

ChUn, M. M., \& JiAnG, Y. (2003). Implicit, long-term spatial contextual memory. Journal of Experimental Psychology: Learning, Memory, \& Cognition, 29, 224-234.

Desimone, R. (1998). Visual attention mediated by biased competition in extrastriate visual cortex. Philosophical Transactions of the Royal Society $B, \mathbf{3 5 3}, 1245-1255$

Desimone, R., \& Duncan, J. (1995). Neural mechanisms of selective visual attention. Annual Review of Neuroscience, 18, 193-222.

DUNCAN, J. (1996). Information and uncertainty in a cumulative science of behavior: 25 years after Broadbent's decision and stress. American Journal of Psychology, 109, 617-655.

DunCAN, J. (1998). Converging levels of analysis in the cognitive neuroscience of visual attention. Philosophical Transactions of the Royal Society $B, \mathbf{3 5 3}, 1307-1317$.

FeCteaU, J. H. (2007). Priming of pop-out depends upon the current goals of observers. Journal of Vision, 7(6, Art. 1), 1-11. doi:10.1167/7.6.1

Fecteau, J. H., \& Munoz, D. P. (2003). Exploring the consequences of the previous trial. Nature Reviews Neuroscience, 4, 435-443.

Folk, C. L., \& Remington, R. W. (2008). Bottom-up priming of topdown attentional control settings. Visual Cognition, 8, 215-231.

Folk, C. L., Remington, R. W., \& Johnston, J. C. (1992). Involuntary covert orienting is contingent on attentional control settings. Journal of Experimental Psychology: Human Perception \& Performance, 18, 1030-1044.

Goolsby, B. A., Grabowecky, M., \& Suzuki, S. (2005). Adaptive modulation of color salience contingent upon global form coding and task relevance. Vision Research, 45, 901-930.

Goolsby, B. A., \& SUZUKI, S. (2001). Understanding priming of colorsingleton search: Roles of attention at encoding and "retrieval." Perception \& Psychophysics, 63, 929-944.

HARTER, M. R., \& Aine, C. J. (1984). Brain mechanisms of visual selective attention. In R. Parasuraman \& D. R. Davies (Ed.), Varieties of attention (pp. 293-321). Orlando, FL: Academic Press.

Hillstrom, A. P. (2000). Repetition effects in visual search. Perception \& Psychophysics, 62, 800-817.

Huang, L., Holcombe, A. O., \& Pashler, H. (2004). Repetition priming in visual search: Episodic retrieval, not feature priming. Memory \& Cognition, 32, 12-20.

LeE, H., \& VecERA, S. P. (2007). Unpublished data.

Lleras, A., KaWahara, J., Wan, X. I., \& Ariga, A. (2008). Intertrial inhibition of focused attention in pop-out search. Perception \& Psychophysics, 70, 114-131.

LuCK, S. J., \& Vogel, E. K. (1997). The capacity of visual working memory for features and conjunctions. Nature, 390, 279-281.

MaljKovic, V., \& NAKaYAma, K. (1994). Priming of pop-out: I. Role of features. Memory \& Cognition, 22, 657-672.

Maljkovic, V., \& Nakayama, K. (1996). Priming of pop-out: II. Role of position. Perception \& Psychophysics, 58, 977-991.

MaljKovic, V., \& NaKayama, K. (2000). Priming of pop-out: III. A short term implicit memory system beneficial for rapid target selection. Visual Cognition, 7, 571-595.

Mozer, M. C., Kinoshita, S., \& Shettel, M. (2007). Sequential dependencies offer insight into cognitive control. In W. Gray (Ed.), Integrated models of cognitive systems (pp. 180-193). New York: Oxford University Press.

Mozer, M. C., Shettel, M., \& Vecera, S. P. (2006). Top-down control of visual attention: A rational account. Advances in Neural Information Processing Systems, 18, 923-930.

Muller, H. J., Heller, D., \& Ziegler, J. (1995). Visual search for singleton feature targets within and across dimensions. Perception \& Psychophysics, 57, 1-17.

Muller, H. J., Reimann, B., \& Krummenacher, J. (2003). Visual 
search for singleton feature targets across dimensions: Stimulusand expectance-driven effects in dimensional weighting. Journal of Experimental Psychology: Human Perception \& Performance, 29, 1021-1035.

Olivers, C. N. L., \& Humphreys, G. W. (2003). Attentional guidance by salient feature singletons depends on intertribal contingencies. Journal of Experimental Psychology: Human Perception \& Performance, 29, 650-657.

Olivers, C. N. L., Meijer, F., \& Theeuwes, J. (2006). Feature-based memory driven attentional capture: Visual working memory content affects visual attention. Journal of Experimental Psychology: Human Perception \& Performance, 32, 1243-1265.

Wolfe, J. M. (1994). Guided Search 2.0: A revised model of visual search. Psychonomic Bulletin \& Review, 1, 202-238.

Wolfe, J. M. (1998). Visual search. In H. Pashler (Ed.), Attention (pp. 13-74). Hove, U.K.: Psychology Press.

Wolfe, J. M., Butcher, S. J., Lee, C., \& Hyle, M. (2003). Changing your mind: On the contributions of top-down and bottom-up guidance in visual search for feature singletons. Journal of Experimental Psychology: Human Perception \& Performance, 29, 483-502.

Wolfe, J. M., Cave, K. R., \& Franzel, S. L. (1989). Guided search: An alternative to the feature integration model for visual search. Journal of Experimental Psychology: Human Perception \& Performance, 15, 419-433.

\section{NOTE}

1. The same patterns of results were found with inclusion of these 8 participants' data. There was a significantly faster response for the target matched with the previous target for color only on the 1-3-back condition $[t(26)=3.0, p<.01]$. However, in the more distant trials, there was no difference between the conditions of previous-current target color matching $[t(26)=1.1, p>.05]$.

(Manuscript received February 18, 2008; revision accepted for publication February 14, 2009.) 